\title{
Fallopian Tube Carcinosarcoma
}

National Cancer Institute

\section{Source}

National Cancer Institute. Fallopian Tube Carcinosarcoma. NCI Thesaurus. Code C40124.

A carcinosarcoma that arises from the fallopian tube. It usually affects postmenopausal women and presents with abdominal pain, abdominal distension or genital bleeding. The prognosis is usually poor. 